\title{
Détection précoce des cancers de la cavité buccale : un outil de formation réalisé par l'Institut national du Cancer
}

\author{
Perreau-Saussine M \\ Institut natianal du Cancer, Boulogne-Billancourt, France \\ mperreau-saussine@institutcancer.fr
}

En 2005, le nombre de nouveaux cas de cancers de la cavité buccale est estimé à 7500 dont trois quarts surviennent chez des hommes ; 1875 décès ont été constatés. Ces cancers touchent essentiellement des hommes de plus de 45 ans, ayant une consommation excessive d'alcool et de tabac.

Globalement en régression, ces cancers restent redoutables avec un taux de survie à 5 ans d'environ $40 \%$. Une des raisons en est qu'ils sont diagnostiqués à un stade trop tardif ce qui alourdit les traitements et diminue les chances de guérison. Or, il est possible par un examen visuel de la cavité buccale de détecter des lésions cancéreuses et pré-cancéreuses. Le chirurgien dentiste peut jouer un rôle majeur dans cette détection et dans l'orientation du patient vers un spécialiste.

La mesure 17 du Plan Cancer, lancé en novembre dernier par le Président de la République, insiste sur l'amélioration des connaissances en matière de détection précoce des cancers et plus particulièrement les cancers de la cavité buccale (action 17.3). Dans ce cadre, l'Institut National du Cancer (INCa) a réalisé un module de formation pour la détection précoce des cancers de la cavité buccale à l'attention des chirurgiens dentistes qui est accessible sur le site internet de l'INCa (www.e-cancer.fr).

Cet outil permet notamment de :

- identifier les populations et les facteurs de risque des cancers de la cavité buccale

- identifier les lésions suspectes à l'examen clinique

- connaitre la conduite à tenir en cas de détection

- disposer des éléments du langage à utiliser avec les patients.

Riche d'une iconographie de plus de 300 images, de vidéos, de cas cliniques et de quiz, cet outil peut aisément être utilisé lors d'une consultation, dans le cadre d'une formation à titre individuel ou comme support de formation lors de présentations. Une version gratuite de l'outil sous forme de CD-Rom est disponible, notamment pour des étudiants des Facultés de Chirurgie dentaire.

Après un exposé relatif à l'épidémiologie des cancers de la cavité buccale, une présentation de l'outil sera proposée aux participants. 